integrating single B cell expression profiling and repertoire analysis, we map the development of $\mathrm{B}$ cells in $\mathrm{BM}$ and peripheral and pathogenic characteristics of early $\mathrm{B}$ cells, especially propre-B.

Conclusion: These findings demonstrated that early B cells in BM, especially propre-B are abnormally differentiated with dysregulations. BM is an important organ targeted by SLE. This studyis not only to clarify the internal mechanism of the disorder of differentiation of B cells, but also to provide new clues for the targeted diagnosis and treatment of SLE.

References:

[1] Palanichamy, A., et al., Neutrophil-mediated IFN activation in the bone marrow alters $B$ cell development in human and murine systemic lupus erythematosus. J Immunol, 2014. 192(3): p. 906-18.

[2] Papadaki, H.A., J.C. Marsh, and G.D. Eliopoulos, Bone marrow stem cells and stromal cells in autoimmune cytopenias. Leuk Lymphoma, 2002. 43(4): p. 753-60.

[3] Karrar, S. and D.S. Cunninghame Graham, Abnormal B Cell Development in Systemic Lupus Erythematosus: What the Genetics Tell Us. Arthritis Rheumatol, 2018. 70(4): p. 496-507.

[4] Woods, M., Y.R. Zou, and A. Davidson, Defects in Germinal Center Selection in SLE. Front Immunol, 2015. 6: p. 425.

[5] Upregulation of p16INK4A promotes cellular senescence of bone marrow-derived mesenchymal stem cells from systemic lupus erythematosus patients. Cell Signal, 2012. 24(12): p. 2307-14.

Disclosure of Interests: None declared

DOI: 10.1136/annrheumdis-2020-eular.6162

\section{THU0045 IDENTIFICATION OF NOVEL CENTROMERE AUTOANTIGENS IN SJÖGREN'S SYNDROME SYSTEMIC SCLEROSIS AND PRIMARY BILIARY CHOLANGITIS.}

N. Kajio ${ }^{1}$, M. Takeshita ${ }^{1}$, K. Suzuki ${ }^{1}$, T. Takeuchi ${ }^{1} .{ }^{1}$ Keio University School of Medicine, Division of Rheumatology, Department of Internal Medicine, Tokyo, Japan

Background: Anti-centromere antibodies (ACA) are detected in the serum of patients with various autoimmune diseases including Sjögren's syndrome (SjS), systemic sclerosis (SSc) and primary biliary cholangitis (PBC). ACA positivity is correlated with clinical manifestations such as Raynaud's phenomenon and sclerodactyly and these features are commonly seen across diseases. Although CENPB is thought to be the major antigen against ACA, autoimmune features of other centromere proteins have not been fully evaluated.

Objectives: The aim of this study is to elucidate centromere autoantigens comprehensively and clarify their association with pathogenesis of SjS, SSc and PBC.

Methods: A centromere protein library was created by cloning 6 single proteins and 10 complexes consisting of 35 proteins belonging to human centromere region. The centromere antigens were immobilized on beads and incubated in the serum of patients with SjS $(n=86)$, SSc $(n=35)$, PBC $(n=10)$, patients with two or more diseases above $(n=44)$, and healthy volunteers $(n=68)$. Autoantibodies to each centromere protein were analyzed by flow cytometry.

Results: Patients had a wide variety of antibodies against most of centromere antigens including 4 newly identified autoantigens. The hierarchical clustering of each antigen distinguished 2 antigen clusters. The reactivity of autoantibodies against a centromere protein of one cluster was mutually correlated regardless of disease types, indicating that these proteins/protein complexes might be the target of ACA. In addition, our method enabled us to detect sera reacted against multiple centromere antigens in some of the ACA-negative patients with existing methods.

Conclusion: We identified 4 novel centromere autoantigens and our data suggested that the main target of ACA was the protein complex rather than a single specific antigen in SjS, SSc and PBC patients. Using the combination of centromere proteins may be useful to detect ACA with higher sensitivity.

References:

[1] Fritzler MJ, Rattner JB, Luft LM, Edworthy SM, Casiano CA, Peebles C, Mahler M. Historical perspectives on the discovery and elucidation of autoantibodies to centromere proteins (CENP) and the emerging importance of antibodies to CENP-F. Autoimmun Rev. 2011;10:194-200.

Disclosure of Interests: Nobuhiko Kajio: None declared, Masaru Takeshita: None declared, Katsuya Suzuki: None declared, Tsutomu Takeuchi Grant/ research support from: Eisai Co., Ltd, Astellas Pharma Inc., AbbVie GK, Asahi Kasei Pharma Corporation, Nippon Kayaku Co., Ltd, Takeda Pharmaceutical Company Ltd, UCB Pharma, Shionogi \& Co., Ltd., Mitsubishi-Tanabe Pharma Corp., Daiichi Sankyo Co., Ltd., Chugai Pharmaceutical Co. Ltd., Consultant of: Chugai Pharmaceutical Co Ltd, Astellas Pharma Inc., Eli Lilly Japan KK, Speakers bureau: AbbVie GK, Eisai Co., Ltd, Mitsubishi-Tanabe Pharma Corporation,
Chugai Pharmaceutical Co Ltd, Bristol-Myers Squibb Company, AYUMI Pharmaceutical Corp., Eisai Co., Ltd, Daiichi Sankyo Co., Ltd., Gilead Sciences, Inc., Novartis Pharma K.K., Pfizer Japan Inc., Sanofi K.K., Dainippon Sumitomo Co., Ltd.

DOI: 10.1136/annrheumdis-2020-eular.1367

\section{THU0046 A PIPELINE TO STUDY ANTIGEN-SPECIFIC CD4+ T CELLS IN RHEUMATOID ARTHRITIS}

R. Kumar ${ }^{1}$, N. Yoosuf ${ }^{1}$, C. Gerstner ${ }^{1}$, S. Turcinov ${ }^{1}$, K. Chemin ${ }^{1}$, V. Malmström ${ }^{1}$ ${ }^{1}$ Karolinska Institutet, Division of Rheumatology, Department of Medicine, Solna, Sweden

Background: Autoimmunity to citrullinated autoantigens forms a critical component of disease pathogenesis in rheumatoid arthritis (RA). Presence of anti-citrullinated protein antibodies (ACPAs) in patients has high diagnostic value. Recently, several citrullinated antigen specific CD4+T cells have been described. However, detailed studies of their T-cell receptor usage and in-vivo profile suffer from the disadvantage that these cells are present at very low frequencies. In this context, we here present a pipeline for TCR repertoire analysis of antigen-specific CD4+T cells from RA patients, including both citrulline and influenza (control) specificities using in-vitro peptide challenge induced-cell expansion.

Objectives: To enable studies of the T cell repertoire of citrullinated antigen-specific CD4+T cells in rheumatoid arthritis

Methods: Peripheral blood mononuclear cells (PBMCs) $(n=7)$ and synovial fluid mononuclear cells (SFMCs) $(n=5)$ from HLA-DR*0401-postive RA patients were cultured in the presence of citrullinated Tenascin $C$ peptide cocktails or influenza peptides (positive control). Citrulline reactive cells were further supplemented with recombinant human IL-15 and IL-7 on day 2. All cultures were replenished with fresh medium on day 6 and rlL-2 was added every 2 days from then. Assessment of proportion of peptide-HLA-tetramer positive cells was performed using flow cytometry whereby individual antigen-specific CD4+T cells were sorted into 96-well plates containing cell lysis buffer, followed by PCR based alpha/beta TCR sequencing. TCR sequencing data was demultiplexed and aligned for TCR gene usage using MiXCR. Some tetramer positive cells were sorted into complete medium containing human IL-2 and PHA for expansion of antigen-specific cells. Cells were supplemented with irradiated allogenic PBMCs (30 times number of antigen specific cells). Clones of antigen specific $\mathrm{CD} 4+\mathrm{T}$ cells were further subjected to tetramer staining to confirm expansion of cells.

Results: As evidenced by increase in frequency of tetramer positive CD4+T cells, in vitro peptide stimulation resulted in expansion of both influenza specific (Fig. 1a) and citrullinated antigen specific (Fig. 1b) CD4+T cells. Polyclonal in-vitro expansion of tenascin $\mathrm{C}$ tetramer positive sorted cells followed by tetramer staining further confirmed antigen specificity and enrichment for antigen specific CD4+T cells after polyclonal stimulation (Fig.1c). TCR repertoire analysis in PB and SF dataset from the first patient showed clonal expansion of influenza specific cells in both sites. Synovial fluid had more diversity of expanding clones as compared to paired PB, with few expanded clones being shared among SF and PB. We observed a more diverse TCR repertoire in citrulline specific CD4+T cells We also observed sharing of TCR alpha chains among different citrulline specific CD4+T cell clones.

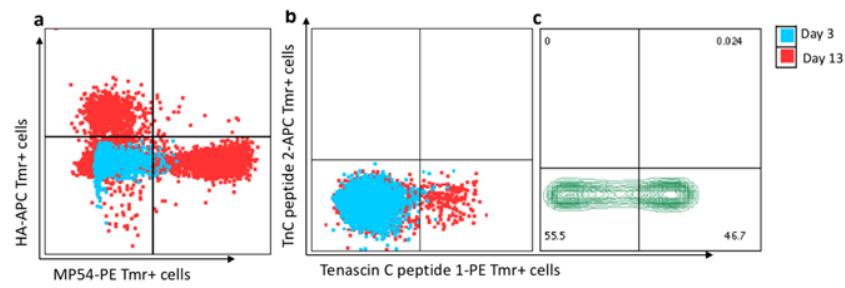

Fig. 1 In-vitro expansion of antigen specific CD4+T cells:

Conclusion: This method provides a highly suitable approach for investigating TCR specificities of antigen specific CD4+T cells under conditions of low cell yields. Building on this dataset will allow us to assess specific features of TCR usage of autoreactive T cells in RA

PBMCs were cultured in presence of (a) influenza (HA, MP54) and (b) citrullinated tenascin peptides. The proportion of antigen specific CD4+T cells was assessed using HLA-class II tetramer staining. We observed an increase in frequency of (a) Infleunza specific cells (red dots in upper left and lower right quadrants) and (b) citrullinated tenascin $\mathrm{C}$ specific cells (red dots in lower right quadrant), at day 13 post culture as compared to day 3. (c) Sorting of citrullinated 
tenascin specific CD4+T cells, followed by $\mathrm{PHA}$ expansion resulted in visible increase in proportion of citrullinated tenascin specific CD4+T cells.

Disclosure of Interests: Ravi kumar: None declared, Niyaz Yoosuf: None declared, Christina Gerstner: None declared, Sara Turcinov: None declared, Karine Chemin: None declared, Vivianne Malmström Grant/research support from: VM has had research grants from Janssen Pharmaceutica DOI: 10.1136/annrheumdis-2020-eular.1093

\section{THU0047 THE SYNOVIUM REWIRES AN IMMUNOLOGICAL RHEOSTAT THAT DEFINES TWO FUNCTIONALLY DISPARATE PATHOGENIC CD4+HLA-DR+ SUBSETS IN HUMAN ARTHRITIS}

J. Y. Leong ${ }^{1}$, P. Kumar ${ }^{1}$, G. Mijnheer ${ }^{2}$, P. Chen ${ }^{1}$, J. G. Yeo ${ }^{1,3}, \mathrm{~S}$ H. Tay ${ }^{1}$, C. Chua ${ }^{1}$, S. N. Hazirah ${ }^{1}$, L. Lai ${ }^{1}$, A. Consolaro ${ }^{4}$, M. Gattorno ${ }^{4}$, T. Arkachaisri ${ }^{1,3}$, A. Martini ${ }^{4}$, F. Van Wijk ${ }^{2}$, S. Albani ${ }^{1} .{ }^{1}$ Translational Immunology Institute, Singhealth/Duke-NUS Academic Medical Centre, Singapore, Singapore; ${ }^{2}$ Laboratory of Translational Immunology, University Medical Center Utrecht, Utrecht University, Utrecht, Netherlands; ${ }^{3}$ Division of Medicine, KK Women's and Children's Hospital, Singapore, Singapore; ${ }^{4}$ Second Paediatric Division, University of Genoa and G Gaslini Institute, Genova, Italy

Background: Despite advances in understanding how the adaptive T cell landscape is affected in human arthritis, specific T cell subset knowledge has yet to be utilised in clinical settings. We have previously discovered within active arthritic patients, a circulating pathogenic-like lymphocyte (CPLs; CD4+HLA-DR+) within the T-effector compartment, that is phenotypically similar to their synovial counterparts. CPLs are inflammatory, correlate with disease activity and overlap in synovial TCR repertoire. A similar inflammation-associated T-regulatory (iaTreg; CD4+HLA-DR+) subset, that is activated, poised to migrate to inflamed site and sharing synovial TCR overlap, suggest a common disease ontogeny that may exist between CPLs and iaTregs.

Objectives: Here we seek to determine whether and how the synovial microenvironment plays a role in modulating these two functionally divergent (Teff/Treg compartments) yet pathogenically homologous subsets. This modulation, akin to an immunological rheostat, may be a feature of the disease process.

Methods: We examined CD45+ immune cells from synovial and PBMCs (active JIA, inactive JIA, paediatric healthy) through mass cytometry (CyToF). CD4 T cells were sorted into CPLs, iaTregs, Teff and Treg through FACS Aria II, from active JIA PBMCs, paired JIA SFMCs and healthy paediatric PBMCs and examined through ngRNASEQ.

Results: Mass cytometric analysis reveal a significant enrichment of synovium signatures in both circulatory CPLs and iaTregs subsets from active arthritic PBMCs, as compared with the conventional pool of Teff/Tregs. This immunological relationship between CPLs/iaTregs is reaffirmed by comparative differential gene expression (DEG) and phylogenetic tree analysis, which indicated transcriptomic convergence between circulatory pathogenic CPLs/iaTreg subsets and divergence from their respective conventional Teff/Treg pools. Circulatory CPLs/iaTregs exhibit (a) common pathway dysregulation in $\mathrm{T}$ cell signalling, (b) restriction in TCR oligoclonality and (c) common transcription factor drivers within the gene regulatory network, suggesting a common pathogenic mechanism acting on these two disparate compartments.

To understand how the microenvironment plays a role in modulating these two subsets, we compared the transcriptome of CPLs/iaTreg and conventional Teff/Treg subsets from (a) healthy PBMCs, (b) JIA PBMCs and (c) paired JIA SFMCs. The convergence between CPLs/iaTreg increases across the spatial/ disease continuum, culminating in 7 key common dysregulated pathways within synovium CPLs/iaTregs. Importantly we detected higher clonotypic sharing of TCRs in CPLs/iaTregs across the spatial and disease continuum, suggesting a common precursor driven by antigenic selection.

Conclusion: Our data suggest that CPLs/iaTregs are dichotomic components of a systemic immune rheostat, shape through the synovium environment, modulating autoimmunity in human arthritis. As iaTreg and CPL most likely have the capacity to morph into each other, the molecular crossroads which control this plasticity represent novel therapeutic targets.

Disclosure of Interests: None declared

DOI: 10.1136/annrheumdis-2020-eular.4006

\section{THU0048 \\ THE FUNCTION CHANGES OF SNPS IN THE P2X7 RECEPTOR BY ALA348TO THR, GLU496TO ALA A ND ARG307TO GLN IN THP-1 CELLS WITH HIGH URIC ACID}

M. Li ${ }^{1}$, T. Jinhui ${ }^{1}$, X. Fang ${ }^{1}$, Y. Ma ${ }^{1}$, X. Pan ${ }^{1}$, X. Dai ${ }^{1}$, X. Li ${ }^{1}$, Y. Wang ${ }^{2}$, X. Li ${ }^{1}$.

${ }^{1}$ The First Affiliated Hospital of USTC, Division of Life Sciences and Medicine,
University of Science and Technology of China, Hefei, China; ${ }^{2}$ Centre for Transplantation and Renal Research, Westmead Institute for Medical Research, The University of Sydney, Sydney, Australia

Background: Previous studies[1] have shown that ATP acts on the sputum receptor P2X ligand-gated ion channel 7 (P2X7R) as a second signal to induce gouty arthritis.

Objectives: In this study, the functional changes of three SNP genotypes, Ala348 to Thr, Glu 496 to Ala and Arg307 to Gln, in P2X7R were analyzed with high uric acid.

Methods: After transfection of HEK-293T cells by lentivirus, observing the uptake ability of HEK-293T cells to ethidium bromide. The effect of three different mutants on the P2X7 receptor was thus observed on the P2X7 channel. In addition, THP-1 cells were also transfected, stable expression of a THP-1 cell line that has been transfected with a wild-type or different mutants and thus established. Then three types were set up separately, and each type was randomized into three groups: MSU(labeled M), MSU+ATP (labeled MA), and unstimulated control group (labeled C).Detection of IL-1 $\beta$ protein expression level in serum by ELISA and NLRP3, ASC and Caspase-1 mRNA levels in transfected THP-1 cells by qRT-PCR.

Results:

1. These three variants have different effects on the uptake function of ATP-induced ethidium ${ }^{+}$bromide in transfection of HEK-293T cells by lentivirus. $\mathrm{Ala}^{348}$ to $\mathrm{Thr}$ increased $\mathrm{P} 2 \mathrm{X}_{7}$-dependent ethidium ${ }^{+}$bromide uptake $(145 \%$ of wild-type $\mathrm{P} 2 \mathrm{X}_{7}$ response, $\left.\mathrm{P}<0.001\right)$. In contrast, Absent or very reduced $\mathrm{P} 2 \mathrm{X}_{7}$ function was found in Glu ${ }^{496}$ to Ala and $\mathrm{Arg}^{307}$ to Gln subjects, appeared to abolish $\mathrm{P} 2 \mathrm{X}_{7}$-dependent dye uptake (38\% and $32 \%$ of wild-type $\mathrm{P} 2 \mathrm{X}_{7}$ responses, $\mathrm{P}<0.001$,), who were compared with wild-type.

2. Compared the IL-1 $\beta$ levels of the three variants with the wide-type and empty virus in THP-1 cells, the $\mathrm{Ala}^{348}$ to Thr mutation significantly up-regulated the serum levels of IL-1 $\beta$ compared with the wide-type and empty virus in group MA with high uric acid $(P=0.0007 ; P=0.013$, respectively). Moreover, similar results have also been shown in IL-1 $\beta$ mRNA expressions $(P=0.0334$; $\mathrm{P}=0.0307$, respectively). The $\mathrm{Glu}^{496}$ to $\mathrm{Ala}$ and $\mathrm{Arg}^{307}$ to Gln mutations down-regulated the serum levels of IL-1 $\beta$ compared with the wide-type in group MA ( $P=0.0189 ; P=0.0164$, respectively).

3. NLRP3 mRNA was significantly increased in the $\mathrm{Ala}^{348}$ to Thr mutation compared with the wide-type and empty virus in group MA ( $p=0.0003 ; P=0.0001$, respectively). However, NLRP3 mRNA was significantly reduced in the $\mathrm{Glu}^{496}$ to Ala and $\mathrm{Arg}^{307}$ to Gln mutations compared with the wide-type in group MA ( $p=0.0294 ; P=0.0279$, respectively).

4. Wild-type was signifcantly higher than empty virus in the ASC gene expression in group $\mathrm{MA}(\mathrm{P}=0.0022)$. Morever, the $\mathrm{Ala}^{348}$ to Thr mutation was higher than empty virus while $\mathrm{Arg}^{307}$ to Gln mutation was lower than that in group MA $(P=0.0138 ; P=0.0283$, respectively).

5. Unlike NLRP3 gene expression, the data showed that the expression of caspase-1 mRNA in group C, M and MA all with no statistical significance, respectively $(P>0.05)$.

Conclusion: Our data revealed that $\mathrm{Ala}^{348}$ to $\mathrm{Thr}$ up-regulate the functional status of P2X7R and Glu ${ }^{496}$ to Ala and $\mathrm{Gln}^{460}$ to Arg down-regulate the functional status of P2X7R, which resulted in a significant increase or decrease in IL-1 $\beta$ and NLRP3 expression levels with high uric acid.

References:

[1] Tao JH, Zhang Y, Li XP, et al. P2X7R: a potential key regulator of acute gouty arthritis[J].Semin Arthritis Rheum, 2013,43(3):376-380.

Disclosure of Interests: None declared

DOI: 10.1136/annrheumdis-2020-eular.1463

\begin{tabular}{|l|l}
\hline THU0049 & DISTINCT EXPRESSION OF COINHIBITORY \\
MOLECULES ON ALVEOLAR T CELLS IN PATIENTS \\
WITH RHEUMATOID ARTHRITIS- AND IDIOPATHIC \\
INFLAMMATORY MYOPATHIES-ASSOCIATED \\
INTERSTITIAL LUNG DISEASE
\end{tabular}

M. Nakazawa ${ }^{1}$, K. Suzuki ${ }^{1}$, M. Takeshita ${ }^{1}$, J. Inamo ${ }^{1}$, H. Kamata ${ }^{2}$, M. Ishii ${ }^{2}$, Y. Oyamada ${ }^{3}$, H. Oshima ${ }^{4}$, T. Takeuchi ${ }^{1}{ }^{1}$ Keio University School of Medicine, Division of Rheumatology, Department of Internal Medicine, Tokyo, Japan; ${ }^{2}$ Keio University School of Medicine, Division of Pulmonary Medicine, Tokyo, Japan; ${ }^{3}$ National Tokyo Medical Center, Department of Respiratory Medicine, Tokyo, Japan; ${ }^{4}$ National Tokyo Medical Center, Department of Connective Tissue Diseases, Tokyo, Japan

Background: Interstitial lung disease (ILD) is a common extra-articular manifestation of rheumatoid arthritis (RA) and also the most common non-musculoskeletal manifestation of idiopathic inflammatory myopathies (IIM), including polymyositis, dermatomyositis and clinically amyopathic dermatomyositis. 